\title{
The Universal Generating Function of Analytical Poisson Structures
}

\section{Journal Article}

Author(s):

Dherin, Benoit

Publication date:

2006-02

Permanent link:

https://doi.org/10.3929/ethz-b-000037337

Rights / license:

In Copyright - Non-Commercial Use Permitted

Originally published in:

Letters in Mathematical Physics 75(2), https://doi.org/10.1007/s11005-005-0034-6 


\title{
The Universal Generating Function of Analytical Poisson Structures
}

\author{
BENOIT DHERIN \\ D-Math,ETH-Zentrum,8092 Zürich, Switzerland.e-mail: dherin@math.ethz.ch
}

Received: 13 May 2005; revised version: 7 November 2005

Published online: 14 January 2006

\begin{abstract}
Generating functions of Poisson structures are special functions which induce, on open subsets of $\mathbb{R}^{d}$, a Poisson structure together with the local symplectic groupoid integrating it. In a previous paper by A. S. Cattaneo, G. Felder and the author, a universal generating function was provided in terms of a formal power series coming from Kontsevich star product. The present article proves that this universal generating function converges for analytical Poisson structures and shows that the induced local symplectic groupoid coincides with the phase space of Karasev-Maslov.
\end{abstract}

\section{Mathematics Subject Classification 58H05 (53D05).}

Key words. symplectic groupoids, generating functions, Poisson manifolds, tree-level knotsevich star product.

\section{Introduction}

Let $U$ be an open subset of $\mathbb{R}^{d}$ and let $S$ be a function defined on a neighborhood of $\{0\} \times\{0\} \times U$ in $\left(\mathbb{R}^{d}\right)^{*} \times\left(\mathbb{R}^{d}\right)^{*} \times U$. In [2] and in [8], it was shown that if $S$ satisfies the SGS conditions and the SGA equation ${ }^{1}$ (see Section 2), then $S$ generates a Poisson structure on $U$ given by

$$
\alpha(x):=\frac{\partial^{2} S}{\partial p_{1} \partial p_{2}}(0,0, x)
$$

together with the local symplectic groupoid integrating it. Its structure maps, defined on a neighborhood $G_{S}(U)$ of the zero section in $T^{*} U$, are given by

$$
\begin{array}{lc}
\epsilon(p, x)=\quad(0, x) & \text { unit map } \\
i(p, x)=\quad(-p, x) & \text { inverse map } \\
s(p, x)=\nabla_{p_{2}} S(p, 0, x) & \text { source map } \\
t(p, x)=\nabla_{p_{1}} S(0, p, x) & \text { target map. }
\end{array}
$$

Suppose now that we have a Poisson structure $\alpha$ on a manifold $M$ and a local chart $U$ with coordinates $x_{1}, \ldots, x_{d}$. We may pose the reciprocal problem of

${ }^{1}$ The SGA equation may be seen as the equation of an associative product in the operad of exact Lagrangian submanifolds of $\left(T^{*} \mathbb{R}^{d}\right)^{k} \times T^{*} \mathbb{R}^{d}$ where the compositions are given by Symplectic reduction. See [3] for a presentation of this operad. 
finding a generating function satisfying SGS and SGA on $U$ such that $\alpha(x):=$ $\frac{\partial^{2} S}{\partial p_{1} \partial p_{2}}(0,0, x)$. Thus, solving this problem for $S$ provides an explicit symplectic realization (in the sense of [14]) of the Poisson manifold $(\alpha, U)$ as well as an explicit description of the unique (up to isomorphism) local symplectic groupoid (see [15]) of $(\alpha, U)$. As a consequence, observe that knowing the generating function $S$ of $\alpha$ on $U$ allows us to transform any Hamilton-Poisson system on $U$

$$
\dot{X}(\tau)=\alpha(X(\tau)) \nabla f(X(\tau)), \quad X(0)=\xi \in U,
$$

where $f \in C^{\infty}(U)$ into the following Hamilton system on $G_{S}(U) \subset T^{*} U$

$$
\begin{aligned}
& \dot{p}(\tau)=-\nabla_{x} H_{f}(p(\tau), q(\tau)) \\
& \dot{q}(\tau)=\nabla_{p} H_{f}(p(\tau), q(\tau)),
\end{aligned}
$$

with Hamiltonian $H_{f}=-f \circ t$. The components of the source map $s^{1}, \ldots, s^{d}$ are $d$ constants of motions.

In fact, for any Poisson structure $\alpha$ and any local chart $U$, we may find a universal formal generating function for $\alpha$. This is the main result of [2]. This universal generating function turns out to be the semi-classical part of Kontsevich star product associated to $\alpha$ on $U$,

$$
S_{\epsilon}(\alpha)\left(p_{1}, p_{2}, x\right)=\left(p_{1}+p_{2}\right) x+\sum_{n=1}^{\infty} \frac{\epsilon^{n}}{n !} \sum_{\Gamma \in T_{n, 2}} W_{\Gamma} \hat{B}_{\Gamma}\left(p_{1}, p_{2}, x\right) .
$$

This relates then symplectic groupoids with star products ${ }^{2}$ as was already foreseen by Coste et al. in [6]. This universal formula is however not a "true" generating function in the sense that it is a formal power series in a formal parameter $\epsilon$. The main result of this article is the following theorem which states that the universal generating function converges for analytical Poisson structures and that the induced local symplectic groupoid coincides with the construction of KarasevMaslov [12].

THEOREM 1. Consider a Poisson manifold $M$ with Poisson bivector $\alpha$ such that for any local chart $U$ and any multi-index $\beta=\left(\beta_{1}, \ldots, \beta_{d}\right) \in \mathbb{N}^{d}$

$$
\left|\partial^{\beta} \alpha^{i j}(x)\right| \leqslant M_{U}(x)^{|\beta|+1},
$$

where $M_{U}(x)$ a positive function.

Then, the universal generating function

$$
S_{\epsilon}(\alpha)\left(p_{1}, p_{2}, x\right)=\left(p_{1}+p_{2}\right) x+\sum_{n=1}^{\infty} \frac{\epsilon^{n}}{n !} \sum_{\Gamma \in T_{n, 2}} W_{\Gamma} \hat{B}_{\Gamma}\left(p_{1}, p_{2}, x\right),
$$

${ }^{2}$ Since then, Karabegov [9] has shown how to associate a formal symplectic groupoid to a general star product on a Poisson manifold. His approach differs essentially from ours by the fact that he defines a formal symplectic groupoid in terms of the functions on it. 
converges absolutely for $\epsilon \in[0,1)$ and

$$
\left\|p_{i}\right\| \leqslant \frac{1}{64 \mathrm{e} M_{U}^{2}(x)}, \quad i=1,2 .
$$

Moreover, the local symplectic groupoid induced by $S_{\epsilon}$ on $U$ is exactly the same not only isomorphic - as the one constructed by Karasev and Maslov [12]. In particular, if we consider the Poisson-Hamilton system

$$
\dot{X}(\tau)=\alpha(X(\tau)) p .
$$

for small $p \in\left(\mathbb{R}^{d}\right)^{*}$, then the end-points of a solution $X$ satisfy $X(0)=s(p, q)$ and $X(1)=t(p, q)$ for $q=\nabla_{p_{2}} S(-p, p, X(0))=\nabla_{p_{1}} S(p,-p, X(1))$.

The proof of this theorem is split between Propositions 1, 2 and 4. Note that the identification with Karasev local symplectic groupoid on $U$ allows us to patch all the induced groupoids in the local charts into a global manifold $G(U)$, which is the local groupoid integrating $(M, \alpha)$. The question of defining a global generating function will be addressed elsewhere.

Let us conclude this introduction by mentioning the article [7] which also deals with generating functions of symplectic groupoids when the base space is a symmetric symplectic space. In this context, the generating function is related to the symplectic area of some geodesic triangles. It would be very interesting to see if the universal generating function also admits such a geometrical interpretation in the general case.

This letter is organized as follows. In Section 1, we briefly review standard results on Poisson manifolds and local symplectic groupoids. Section 2 introduces the notion of generating functions of Poisson structures and provides examples. In Section 3, we prove the convergence of the universal generating function for analytical Poisson structures. In Section 4, we compare the local symplectic groupoid generated by this convergent generating function and the one constructed by Maslov and Karasev [12].

The results presented here are part of the author's $\mathrm{PhD}$ thesis [8] and may be seen as a natural development of [2].

\section{Lie System and Local Symplectic Groupoids}

In this section, we present a system of non-linear PDEs on an open subset $U$ of $\mathbb{R}^{d}$ (that we called the reduced Lie system), and we briefly describe how a solution of this system generates a local symplectic groupoid structure over $U$. We also explain how any solution of the reduced Lie system may be used to transform a Poisson-Hamilton system on $U$ into a Hamilton system on the local symplectic groupoid. The results stated here were established by Coste et al. [6], Karasev [10,11], Karasev and Maslov [12] and Weinstein [14]. Therefore, we refer the reader to these articles for details and proofs. 
The reduced Lie system. Let $U$ be an open subset of $\mathbb{R}^{d}$ and $V$ be an open neighborhood of $U$ in $T^{*} U$. We say that the functions $s^{i}: V \rightarrow \mathbb{R}, i=1, \ldots, d$ are a solution of the reduced Lie system if for all $i, j=1, \ldots, d$

$$
\sum_{u=1}^{d} \frac{\partial s^{i}}{\partial x_{u}} \frac{\partial s^{j}}{\partial p^{u}}-\frac{\partial s^{i}}{\partial p_{u}} \frac{\partial s^{j}}{\partial x^{u}}=\alpha^{i j}\left(s^{1}, \ldots, s^{d}\right),
$$

for some functions $\alpha^{i j}: U \rightarrow \mathbb{R}, i, j=1, \ldots, d$ and the $s=\left(s^{1}, \ldots, s^{d}\right)$ satisfy the initial condition $s(0, x)=x$ and the non-degeneracy condition

$$
\operatorname{det}\left(\nabla_{x} s(p, x)\right) \neq 0
$$

Remark 1.

1. A necessary condition for the existence of a solution is that the matrix $\alpha(x)=\left(\alpha^{i j}(x)\right)_{i, j=1}^{d}$ is a Poisson structure on $U$.

2. If we denote by $\{,\}_{\alpha}$ the Poisson bracket on $U$ associated to $\left(\alpha^{i j}(x)\right)_{i, j=1}^{d}$ and by $\{,\}_{\omega}$ the Poisson bracket associated to the canonical symplectic form $\omega=d p \wedge d q$ on $V \subset T^{*} U$, then the reduced Lie system may be written as

$\left\{s^{i}, s^{j}\right\}_{\omega}=\alpha^{i j}(s), \quad i, j=1, \ldots, d$.

It implies that $s=\left(s^{1}, \ldots, s^{d}\right)$ is a Poisson map from $(V, \omega)$ to $(U, \alpha)$, i.e., that

$\left\{s^{*} f, s^{*} g\right\}_{\omega}=s^{*}\{f, g\}_{\alpha}$.

for all $f, g \in C^{\infty}(U)$. Such a map is also called a symplectic realization of the Poisson manifold $(U, \alpha)$.

3. Suppose that $s$ is a solution of the reduced Lie system. The initial and non-degeneracy conditions imply, by the implicit function theorem, that there exists an inverse $Q$ such that

$$
Q(p, s(p, q))=q \quad \text { and } \quad s(p, Q(p, x))=x .
$$

Hamiltonian lift. Let $\alpha$ be a Poisson structure on $U \subset \mathbb{R}^{d}$ and let $s$ be a solution of the reduced Lie system. An easy consequence of the fact that $s$ is a Poisson map from $(V, \omega)$ to $(U, \alpha)$ is the following. The Hamilton-Poisson system on $U$ with Hamiltonian $f \in C^{\infty}(U)$, i.e.,

$$
\dot{X}(\tau)=\alpha^{i j}(X(\tau)) \partial_{j} f(X(\tau)), \quad X(0)=\xi \in U,
$$


may be lifted to the Hamilton system on $V$

$$
\begin{aligned}
& \dot{p}(\tau)=-\nabla_{x} H_{f}(p(\tau), q(\tau)) \\
& \dot{q}(\tau)=\nabla_{p} H_{f}(p(\tau), q(\tau)),
\end{aligned}
$$

with Hamiltonian $H_{f}=f \circ s$. In other words, if $(p(\tau), q(\tau))$ is the solution of the Hamilton system on $V$, then $X(\tau):=s(p(\tau), q(\tau))$ is the solution of the Poisson system on $U$ provided that $s(p(0), q(0))=\xi$. This Hamilton system possesses $d$ integrals of the motion. Let us construct them as done in [11]. Consider on $U$ the time-dependent Hamiltonian $K(x, t)=\langle Q(-p t, x), p\rangle$. Denote by $\phi_{K}^{\tau}$ the flow generated by $K$ and define

$$
t(p, q):=\left.\phi_{K}^{\tau}(s(p, q))\right|_{\tau=1} .
$$

In [11], it is shown that $t=\left(t^{1}, \ldots, t^{d}\right)$ is the only map such that

$$
\begin{aligned}
\left\{t^{i}, t^{j}\right\}_{\omega}(p, x) & =-\alpha^{i j}(t(x, p)), \\
\left\{s^{i}, t^{j}\right\}_{\omega}(p, x) & =0 \\
t(0, x) & =x \text { and } \operatorname{det}\left(\nabla_{x} t(p, x)\right) \neq 0 .
\end{aligned}
$$

Thus, we may easily prove from (3) that $t=\left(t^{1}, \ldots, t^{d}\right)$ constitute $d$ constants of the motion of the Hamilton system on $V$. The system of PDEs defined by Equations (1), (2) and (3) together with the respective initial and non-degeneracy conditions for $s$ and $t$ is called the Lie system.

Local groupoid structure. We may now describe the local symplectic groupoid over $U$ generated by a solution of the Lie system. Suppose that $(s, t)$ is a solution of the Lie system defined on a open neighborhood $\mathrm{G}_{\mathrm{U}}(s, t)$ of $U$ in $T^{*} U$. Thus, $\mathrm{G}_{\mathrm{U}}(s, t)$ inherits from $T^{*} U$ the canonical symplectic form $\mathrm{d} p \wedge \mathrm{d} q$ turning it into a symplectic manifold. This will be the local symplectic groupoid over $U$ associated to a solution of the Lie system. The structure maps are namely given by $s$ for the source, by $t$ for the target and by $\epsilon(x)=(0, x)$ for the unit $\epsilon: U \rightarrow \mathrm{G}_{\mathrm{U}}(s, t)$. The crucial point is to define the product and the inverse in the following way. Take $(p, x) \in \mathrm{G}_{\mathrm{U}}(s, t)$. If $p$ is small enough, it is always possible to find a time-dependent Hamiltonian $f_{\tau} \in C^{\infty}(U), \tau \in \mathbb{R}_{+}$, such that the flow $\Psi_{H}^{\tau}$ of its Hamiltonian lift $H=-f_{\tau} \circ t \in C^{\infty}\left(\mathrm{G}_{\mathrm{U}}(s, t)\right)$ carries $(0, s(p, x))$ to $(p, x)$ in time $\tau=1$. Then, the inverse of $(p, x)$ is defined as

$$
i(p, x)=\left(\Psi_{H}^{\tau}\right)_{\tau=1}^{-1}(0, t(p, x)),
$$

and for any $(\bar{p}, \bar{x})$ such that $s(p, x)=t(\bar{p}, \bar{x})$, the product between the two points is defined as

$$
((p, x),(\bar{p}, \bar{x})):=\left.\Psi_{H}^{\tau}((\bar{p}, \bar{x}))\right|_{\tau=1} .
$$

This local symplectic groupoid is essentially unique, see for instance [6] and [11] for details and proofs. 


\section{Generating Functions and Local Symplectic Groupoids}

In this section, we consider special functions which induce on an open subset $U$ of $\mathbb{R}^{d}$ both a Poisson structure and the local groupoid which integrates it. As these functions are in fact usual generating functions of some Lagrangian submanifolds, we will call them generating functions of the local symplectic groupoid or of the associated Poisson structure. We will see how such generating functions provide explicit solutions of the Lie system. Let us first recall what we mean by generating functions in the context of cotangent bundle.

DEFINITION 1. (GENERATING FUNCTIONS OF EXACT LAGRANGIAN SUBMANIFOLDS) Consider the cotangent bundle $T^{*} Q$ over a manifold $Q$ endowed with its canonical symplectic form $\omega=-d \theta$. Then every 1 -form $\mu \in \Omega^{1}(Q)$ can be considered as an embedding of $Q$ in $T^{*} Q\left(\mu(Q) \subset T^{*} Q\right)$. This embedding gives a Lagrangian submanifold when $\mu^{*} \omega=0$, i.e., when $\mu$ is closed (namely $\mu^{*} \omega=$ $\left.-\mu^{*} d \theta=-d \mu^{*} \theta=-d \mu\right)$ ). We can then associate to each closed one-form a Lagrangian submanifold of $T^{*} Q$. Moreover when $\mu$ is exact $\mu=d S, S \in C^{\infty}(Q)$ we call $S$ the generating function of $\mu(Q)$.

We introduce some notations. Let $U \subset \mathbb{R}^{d}$ be an open set. Then set

$$
\begin{aligned}
B_{n}(U) & :=\left(\mathbb{R}^{d^{*}}\right)^{n} \times U, \quad B_{n}^{0}(U):=\{0\}^{n} \times U \\
B_{n} & :=B_{n}\left(\mathbb{R}^{d}\right), \quad B_{n}^{0}:=B_{n}^{0}\left(\mathbb{R}^{d}\right) .
\end{aligned}
$$

DEFINITION 2 (SGA AND SGS). Let $U \subset \mathbb{R}^{d}$ be an open subset. We say that a function $S \in C^{\infty}\left(B_{2}\right)$ satisfies the Symplectic Groupoid Associativity $(S G A)$ equation ${ }^{3}$ on $U$ if for each $\left(p_{1}, p_{2}, p_{3}, x\right) \in B_{3}(U)$ sufficiently close from $B_{3}^{0}(U)$ the following implicit equation for $\bar{x}, \bar{p}, \tilde{x}$ and $\tilde{p}$,

$$
\begin{array}{ll}
\bar{x}=\nabla_{p_{1}} S\left(\bar{p}, p_{3}, x\right), & \bar{p}=\nabla_{x} S\left(p_{1}, p_{2}, \bar{x}\right), \\
\tilde{x}=\nabla_{p_{2}} S\left(p_{1}, \tilde{p}, x\right), & \tilde{p}=\nabla_{x} S\left(p_{2}, p_{3}, \tilde{x}\right),
\end{array}
$$

has an unique solution and if the following additional equation holds

$$
S\left(p_{1}, p_{2}, \bar{x}\right)+S\left(\bar{p}, p_{3}, x\right)-\bar{x} \bar{p}=S\left(p_{2}, p_{3}, \tilde{x}\right)+S\left(p_{1}, \tilde{p}, x\right)-\tilde{x} \tilde{p} .
$$

\footnotetext{
${ }^{3}$ The SGA equation may also be written as

$$
\operatorname{Stat}_{(\bar{p}, \bar{x})}\left\{S\left(p_{1}, p_{2}, \bar{x}\right)+S\left(\bar{p}, p_{3}, x\right)-\bar{x} \bar{p}\right\}=\operatorname{Stat}_{(\tilde{p}, \tilde{x})}\left\{S\left(p_{2}, p_{3}, \tilde{x}\right)+S\left(p_{1}, \tilde{p}, x\right)-\tilde{x} \tilde{p}\right\}
$$
}

where $\operatorname{Stat}_{x}\{f(x)\}$ denote the value of the function $f$ at the "stationary" point $\bar{x}$, i.e., the point $\bar{x}$ such that $\nabla f(\bar{x})=0$. Note that we require the stationary points $(\bar{p}, \bar{x})$ and $(\tilde{p}, \tilde{x})$ to be unique in order the definition to make sense. 
Moreover, if $S(p, 0, x)=S(0, p, x)=p x$ and $S(p,-p, x)=0$, we say that $S$ satisfies the symplectic groupoid structure ( $S G S$ ) conditions. At last, a function $S \in$ $C^{\infty}\left(B_{2}\right)$ satisfying both the $S G A$ equation and the $S G S$ conditions will be called a symplectic groupoid generating function on $U$ or simply, when no ambiguities arise, a generating function.

Let $S$ be a generating function on $U$. We define the phase space of $S$ on $U$ by

$$
\mathrm{G}_{\mathrm{U}}(\mathrm{S}):=\left\{(p, x) \in T^{*} U: \nabla_{p_{1}} S(0, p, x) \in U \quad \text { and } \quad \nabla_{p_{2}} S(p, 0, x) \in U\right\} .
$$

It is easy to see from the SGS conditions that $\mathrm{G}_{\mathrm{U}}(\mathrm{S})$ is an neighborhood of the zero section in $T^{*} U$. Thus, $\mathrm{G}_{\mathrm{U}}(\mathrm{S})$ inherits the canonical symplectic structure of $T^{*} U$, turning $\mathrm{G}_{\mathrm{U}}(\mathrm{S})$ into a symplectic manifold. We proved in [2] and [8] that the matrix

$$
\alpha(x)=\left(2 \nabla_{p_{k}^{1}} \nabla_{p_{l}^{2}} S(0,0, x)\right)_{k, l=1}^{d}
$$

is a Poisson structure on $U$. For this reason, we call $S$ the generating function of the Poisson structure $\alpha$. Moreover, the maps defined by

$$
s(p, x)=\nabla_{p_{2}} S(p, 0, x) \quad \text { and } \quad t(p, x)=\nabla_{p_{1}} S(0, p, x)
$$

constitute a solution of the Lie system on $(U, \alpha)$. In particular $s$ is a symplectic realization of the Poisson manifold $(U, \alpha)$, i.e., a Poisson map from the symplectic manifold $\left(G_{S}(U), \mathrm{d} p \wedge \mathrm{d} q\right)$ into the Poisson manifold $(U, \alpha)$. The local inverses of $s$ and $t$ are given by the explicit formulas

$$
Q(p, x)=\nabla_{p_{2}} S(-p, p, x) \quad \text { and } \quad \tilde{Q}(p, x)=\nabla_{p_{1}} S(p,-p, x) .
$$

Finally, summarizing the facts above, we have shown that $G_{S}(U)$ is the local symplectic groupoid integrating $\alpha$ with target $t$, source $s$, unit $\epsilon(x)=(0, x)$ and inverse $i(p, x)=(-p, x)$. The multiplication space $G_{S}(U)^{(m)}$ is the graph of $\mathrm{d} S$.

Let us go through a series of examples.

EXAMPLE 1. The function $S\left(p_{1}, p_{2}, x\right)=\left\langle x, \mu\left(p_{1}, p_{2}\right)\right\rangle$ where $\mu: \mathbb{R}^{d^{*}} \times \mathbb{R}^{d^{*}} \rightarrow \mathbb{R}^{d^{*}}$ is a bilinear associative map on $\mathbb{R}^{d^{*}}$ always satisfies the SGA equation. We get immediately that

$$
\bar{x}=\left\langle x, \nabla_{p_{1}} \mu\left(\tilde{p}, p_{2}\right)\right\rangle, \quad \bar{p}=\mu\left(p_{1}, p_{2}\right), \quad \tilde{x}=\left\langle x, \nabla_{p_{2}} \mu\left(p_{2}, \tilde{p}\right)\right\rangle, \quad \tilde{p}=\mu\left(p_{2}, p_{3}\right) .
$$

An easy computation shows that $S$ satisfies the SGA equation iff

$$
\mu\left(p_{1}, \mu\left(p_{2}, p_{3}\right)\right)=\mu\left(\mu\left(p_{1}, p_{2}\right), p_{3}\right) .
$$

The matrix

$$
\nabla_{p^{1}} \nabla_{p^{2}} S(0,0, x)=\left(2 x^{i} \frac{\partial \mu_{i}}{\partial p_{k}^{1} \partial p_{l}^{2}}(0,0, x)\right)_{k, l=1}^{d}
$$


is a Poisson structure if $\mu$ satisfies

$$
\mu(p, 0)=\mu(0, p)=p \quad \text { and } \quad \mu(p,-p)=\mu(-p, p)=0 .
$$

Notice that it is possible to find a function $S \in C^{\infty}\left(B_{2}\right)$ which satisfies the SGA equation but not the SGS conditions.

EXAMPLE 2. Consider $T^{*} \mathbb{R}^{2}$ and identify $\mathbb{R}^{2 *}$ with the complex plane. We define $S$ as above with $\mu\left(p_{1}, p_{2}\right)=p_{1} p_{2}$ induced by the complex multiplication. Thus,

$$
S\left(\left(p_{x}^{1}, p_{y}^{1}\right),\left(p_{x}^{2}, p_{y}^{2}\right),(x, y)\right)=x\left(p_{x}^{1} p_{x}^{2}-p_{y}^{1} p_{y}^{2}\right)+y\left(p_{x}^{1} p_{y}^{2}+p_{y}^{1} p_{x}^{2}\right)
$$

satisfies the SGA equation but not the SGS conditions. By the way the matrix

$$
\nabla_{p^{1}} \nabla_{p^{2}} S(0,0, x)=\left(\begin{array}{cc}
x & y \\
y & -x
\end{array}\right)
$$

is not a Poisson matrix.

EXAMPLE 3. Let $\mu: \mathbb{R}^{d^{*}} \times \mathbb{R}^{d^{*}} \rightarrow \mathbb{R}^{d^{*}}$ be the vector addition. Then, this time, $S\left(p_{1}, p_{2}, x\right)=x\left(p_{1}+p_{2}\right)$ satisfies not only the SGA equation but also the SGS conditions. The associate structure of symplectic groupoid is the trivial one. The structure maps are $s(p, x)=x, t(p, x)=x, \epsilon(x)=(0, x) i(p, x)=(-p, x)$. The composition is the fiberwise addition. Moreover the induced Poisson structure on the base is the null Poisson structure.

EXAMPLE 4. Consider $S\left(p_{1}, p_{2}, x\right)=x\left(p_{1}+p_{2}\right)+\epsilon \alpha^{i j} p_{i}^{1} p_{j}^{2}$ where $\left(\alpha^{i j}\right)_{i, j=1}^{d}$ is a matrix. We get that

$$
\bar{x}^{l}=x^{l}+\epsilon \alpha^{l j} p_{j}^{3}, \quad \bar{p}_{l}=p_{l}^{1}+p_{l}^{2}, \quad \tilde{x}^{l}=x^{l}+\epsilon \alpha^{i l} p_{i}^{1}, \quad \tilde{p}_{l}=p_{l}^{2}+p_{l}^{3} .
$$

One sees easily that $S$ satisfies the SGA equation for any matrix $\left(\alpha^{i j}\right)_{i, j=1}^{d}$. Moreover,

$$
\nabla_{p^{1}} \nabla_{p^{2}} S(0,0, x)=\left(\alpha^{i j}\right)_{i, j=1}^{d} .
$$

Imposing the SGS conditions to $S$ is equivalent to say that the matrix $\left(\alpha^{i j}\right)_{i, j=1}^{d}$ is skew-symmetric, which also imply that $\nabla_{p^{1}} \nabla_{p^{2}} S(0,0, x)$ is a Poisson structure of constant rank. The multiplication space can then be described as

$$
G_{S}^{m}=\left\{\left(\left(p_{1}, x+\epsilon \alpha p_{2}\right),\left(p_{2}, x-\epsilon \alpha p_{1}\right),\left(p_{1}+p_{2}, x\right)\right),\left(p_{1}, p_{2}, x\right) \in B_{2}\right\} .
$$

The symplectic groupoid structure maps are given by

$$
\begin{aligned}
\epsilon(x) & =(0, x) \\
i(p, x) & =(-p, x) \\
s(p, x) & =x-\epsilon \alpha p \\
t(p, x) & =x+\epsilon \alpha p .
\end{aligned}
$$


EXAMPLE 5. Suppose $\mathbb{R}^{d^{*}}$ is given the structure of a Lie algebra, i.e., there is a Lie bracket [, ]: $\mathbb{R}^{d^{*}} \times \mathbb{R}^{d^{*}} \rightarrow \mathbb{R}^{d^{*}}$. In components we have $\left[p^{1}, p^{2}\right]_{k}=\alpha_{k}^{i j} p_{i}^{1} p_{j}^{2}$ where the $\alpha_{k}^{i j}$,s are the structure constants of the Lie algebra. The Baker-CampellHausdorff formula

$$
C B H\left(p_{1}, p_{2}\right)=p_{1}+p_{2}+\frac{1}{2}\left[p_{1}, p_{2}\right]+\frac{1}{12}\left(\left[p_{1},\left[p_{2}, p_{2}\right]\right]+\left[p_{2},\left[p_{2}, p_{1}\right]\right]\right)+\cdots,
$$

induces a structure of group law in a neighborhood of 0 in $\mathbb{R}^{d *}$. As in Example 1, define

$$
S_{\epsilon}\left(p_{1}, p_{2}, x\right)=\left\langle x, \frac{1}{\epsilon} C B H\left(\epsilon p_{1}, \epsilon p_{2}\right)\right\rangle .
$$

$S$ satisfies the SGA equation because of the associativity of CBH. Moreover, $S$ satisfies also the SGS conditions. The induced Poisson structure on $\mathbb{R}^{d}$ is

$$
\nabla_{p^{1}} \nabla_{p^{2}} S(0,0, x)=\left(\alpha_{k}^{i j} x^{k}\right)_{i, j=1}^{d} .
$$

Notice that, in this case, the induced Poisson structure is linear.

\section{The Universal Generating Function}

A generating function $S$ produces a Poisson structure on $U$ together with the local symplectic groupoid integrating it. In [2], the reciprocal problem was formally solved. Namely, for every Poisson structure $\alpha$ on an open subset $U$ of $\mathbb{R}^{d}$, we may find a formal power series which is formally a generating function of the Poisson structure. It turns out to be the semi-classical part of the Kontsevich starproduct on $U$. In this section, we will first briefly recall the material necessary to write down this formal generating function, i.e., the Kontsevich graphs, weights and operators (we also refer the reader to the original article [13]). Then, we will show the convergence of this generating function for analytical Poisson structures giving thus a true generating function, as well as an explicit solution of the Lie system.

\subsection{KONTSEVICH GRAPHS, WEIGHTS AND OPERATORS}

A Kontsevich graph $\Gamma$ of type $(n, m)$ is a directed graph $\Gamma=\left(E_{\Gamma}, V_{\Gamma}\right)$ which has the following additional structures:

- It possesses two types of vertices $V_{\Gamma}=V_{\Gamma}^{a} \sqcup V_{\Gamma}^{g}$, the aerial vertices $V_{\Gamma}^{a}=\{1, \ldots, n\}$ and the ground vertices $V_{\Gamma}^{g}=\{\overline{1}, \ldots, \bar{m}\}$.

- Each aerial vertex possesses exactly two ordered edges starting from it. The edge set can be described as $E_{\Gamma}=\left\{\left(k, \gamma^{i}(k)\right), \quad k=1, \ldots, n, \quad i=1,2\right\}$ where $\gamma^{i}: V_{\Gamma}^{a} \rightarrow V_{\Gamma}, i=1,2$ (sometimes we denote the two edges of a vertex $k$ by $e_{k}^{1}$ and $e_{k}^{2}$ ). 
- For each aerial vertex $v$ we do not allow small loops (i.e., that $\left.\gamma^{i}(v)=v\right)$ and double edges (i.e., that $\gamma^{1}(v)=\gamma^{2}(v)$ ).

We denote the set of Kontsevich graphs of type $(n, m)$ by $G_{n, m}$ and we set $|\Gamma|:=n$ for $\Gamma \in G_{n, m}$. Let $\Delta(\Gamma)=\left(V_{\Gamma}^{a}, E_{\Gamma}^{a}\right)$ be the restriction of $\Gamma \in G_{n, m}$ to the aerial vertices. The legs of $\Gamma$ are the edges defined by $E_{\Gamma}^{g}=E_{\Gamma} \backslash E_{\Gamma}^{a}$. Thus, $\Delta(\Gamma)$ is $\Gamma$ without its ground vertices and legs. We say that a Kontsevich graph $\Gamma$ is connected if $\Delta(\Gamma)$ is connected in the usual sense. The connected components of a Kontsevich graph $\Gamma$ are the usual connected components of $\Delta(\Gamma)$ to which we add the legs back. We say that a connected Kontsevich graph $\Gamma$ is a Kontsevich tree if $\Delta(\Gamma)$ is a tree (i.e., a connected graph without cycle). Denote by $T_{n, m}$ the set of Kontsevich trees of type $(n, m)$.

Given a Poisson structure $\alpha$ on $\mathbb{R}^{d}$, we may associate to each graph $\Gamma \in G_{n, m}$ an $m$-multidifferential operator on $C^{\infty}\left(\mathbb{R}^{d}\right)$. The general formula is the following

$$
\begin{aligned}
B_{\Gamma}\left(f_{1} \ldots, f_{m}\right):= & \sum_{I: E_{\Gamma} \rightarrow\{1, \ldots, d\}}\left[\prod_{k \in V_{\Gamma}^{a}}\left(\prod_{\substack{e \in E_{\Gamma} \\
e=(*, k)}} \partial_{I(\mathrm{e})}\right) \alpha^{I\left(\mathrm{e}_{k}^{1}\right) I\left(\mathrm{e}_{k}^{2}\right)}\right] \times \\
& \times \prod_{i \in V_{\Gamma}^{g}}\left(\prod_{\substack{e \in E_{\Gamma} \\
e=(* i)}} \partial_{I(e)}\right) f_{i} .
\end{aligned}
$$

We call $\hat{B}_{\Gamma}$ the symbol of $B_{\Gamma}$. It may be defined by the formula ${ }^{4}$

$$
B_{\Gamma}\left(\mathrm{e}^{p_{1} x}, \ldots, \mathrm{e}^{p_{m} x}\right)=\hat{B}_{\Gamma}\left(p_{1}, \ldots, p_{m}, x\right) \mathrm{e}^{\left(p_{1}+\cdots+p_{m}\right) x} .
$$

EXAMPLE 6. Consider the Kontsevich graph of type $(n, 2)$ in Figure 1. The Kontsevich bidifferential operator associated to it is given by

$$
B_{\Gamma}(f, g)(x)=\sum_{1 \leq i, j, k, l, m, n \leq d} \alpha^{i j}(x) \partial_{n} \partial_{j} \alpha^{k l}(x) \alpha^{m n}(x) \partial_{k} f(x) \partial_{i} \partial_{l} \partial_{m} g(x) .
$$

As for the symbol, we have

$$
\hat{B}_{\Gamma}\left(p_{1}, p_{2}, x\right)=\sum_{1 \leq i, j, k, l, m, n \leq d} \alpha^{i j}(x) \partial_{n} \partial_{j} \alpha^{k l}(x) \alpha^{m n}(x) p_{k}^{1} p_{i}^{2} p_{l}^{2} p_{m}^{2} .
$$

Associated to each Kontsevich graph $\Gamma \in G_{n, m}$, there is also a number, the Kontsevich weight $W_{\Gamma}$. As the graphs involved in the expression of the formal generating function are of type $(n, 2)$, we define the weights only for these graphs.

\footnotetext{
${ }^{4}$ We use the notation $p x$ to mean the sum $\sum_{i=1}^{d} p_{i} x_{i}$ for $x \in \mathbb{R}^{d}$ and $p \in\left(\mathbb{R}^{d}\right)^{*}$.
} 


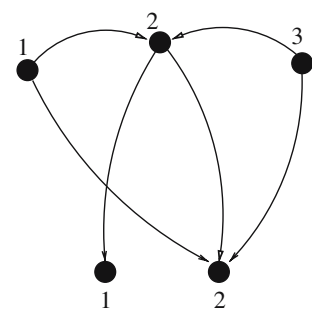

Figure 1. A Kontsevich graph $\Gamma$ of type $(n, 2)$.

(it makes things easier to write but the generalization is however straightforward). To begin with, consider the set

$$
M_{n}:=\left\{\left(z_{1}, \ldots, z_{n}\right): z_{i} \in \mathcal{H} \text { and } z_{i} \neq z_{j} \text {, for } i, j=1, \ldots, n\right\},
$$

where $\mathcal{H}$ is the hyperbolic upper half complex plane. $M_{n}$ is a real non-compact manifold of dimension $2 n$. It may be compactified into a compact manifold with corners $\overline{M_{n}}$ in which $M_{n}$ is an open stratum. In this short exposition, we will avoid to discuss the compactification issues and do as if $M_{n}$ were compact. Constructions, details and proofs may be found in [13]. For each graph $\Gamma \in G_{n, 2}$, we construct a map $\psi_{\Gamma}$ from $M_{n}$ into $\left(S^{1} \times S^{1}\right)^{n}$ in the following way. We set

$$
\begin{aligned}
\psi_{\Gamma}\left(z_{1}, \ldots, z_{n}\right)= & \left(\phi^{h}\left(z_{1}, z_{\gamma^{1}(1)}\right), \phi^{h}\left(z_{1}, z_{\gamma^{2}(1)}\right), \ldots\right. \\
& \left.\ldots, \phi^{h}\left(z_{n}, z_{\gamma^{1}(n)}\right), \phi^{h}\left(z_{n}, z_{\gamma^{2}(n)}\right)\right) .
\end{aligned}
$$

where

$$
\phi^{h}\left(z_{1}, z_{2}\right):=\frac{1}{2 i} \ln \frac{z_{1}-z_{2}}{\bar{z}_{1}-z_{2}} \frac{z_{1}-\bar{z}_{2}}{\bar{z}_{1}-\bar{z}_{2}}
$$

is the hyperbolic angle between the geodesic ${ }^{5}$ passing through $z_{1}$ and $z_{2}$ and the geodesic perpendicular to the real axis and passing through $z_{1}$ in the hyperbolic plane $\mathcal{H} .\left(S^{1} \times S^{1}\right)^{n}$ is endowed with a canonical volume form

$$
\omega_{V}=\frac{1}{(2 \pi)^{n}} \mathrm{~d} \phi_{1}^{1} \wedge \mathrm{d} \phi_{1}^{2} \wedge \cdots \wedge \mathrm{d} \phi_{n}^{1} \wedge \mathrm{d} \phi_{n}^{2},
$$

where $\phi_{1}^{1}, \phi_{1}^{2}, \ldots, \phi_{n}^{1}, \phi_{n}^{2}$ are the angle coordinates of $\left(S^{1} \times S^{1}\right)^{n}$. The Kontsevich weight of $\Gamma$ is given by integrating the pullback of $\omega_{V}$ by $\psi_{\Gamma}$ on $M_{n}$

$$
W_{\Gamma}:=\int_{M_{n}} \psi_{\Gamma}^{*} \omega_{V} .
$$

At this point, we have all necessary ingredients to write the universal formal generating function associated to a Poisson structure $\alpha$ on a open subset $U$ of $\mathbb{R}^{d}$. It is given by the formula

\footnotetext{
${ }^{5}$ The geodesics of the hyperbolic plane $\mathcal{H}:=\{z \in \mathbb{C}: \Im z>0\}$ are arcs of circles and segments of lines which are perpendicular to the real axis.
} 


$$
S_{\epsilon}(\alpha)\left(p_{1}, p_{2}, x\right)=\left(p_{1}+p_{2}\right) x+\sum_{n=1}^{\infty} \frac{\epsilon^{n}}{n !} \sum_{\Gamma \in T_{n, 2}} W_{\Gamma} \hat{B}_{\Gamma}\left(p_{1}, p_{2}, x\right),
$$

where $x \in U \subset \mathbb{R}^{d}, p_{1}, p_{2} \in\left(\mathbb{R}^{d}\right)^{*}$ and $\epsilon$ is a formal parameter. We refer the reader to [2] for the proof that $S_{\epsilon}(\alpha)$ is a formal generating function of $\alpha$.

\subsection{CONVERGENCE OF THE GENERATING FUNCTION}

We show in the following proposition that $S_{\epsilon}(\alpha)$ converges for $\epsilon \in(0,1)$ and small $p_{1}, p_{2}$ if the Poisson structure $\alpha$ is analytical.

PROPOSITION 1. Consider a Poisson manifold $M$ with Poisson bivector $\alpha$ such that for any local chart $U$

$$
\left|\partial^{\beta} \alpha^{i j}(x)\right| \leqslant M_{U}(x)^{|\beta|+1},
$$

where $\beta=\left(\beta_{1}, \ldots, \beta_{d}\right) \in \mathbb{N}^{d}$ is a multi-index and $M_{U}(x)$ a positive function. Then, the universal generating function

$$
S_{\epsilon}(\alpha)\left(p_{1}, p_{2}, x\right)=\left(p_{1}+p_{2}\right) x+\sum_{n=1}^{\infty} \frac{\epsilon^{n}}{n !} \sum_{\Gamma \in T_{n, 2}} W_{\Gamma} \hat{B}_{\Gamma}\left(p_{1}, p_{2}, x\right),
$$

converges absolutely for $\epsilon \in[0,1)$ and

$$
\left\|p_{i}\right\| \leqslant \frac{1}{64 \mathrm{e} M_{U}^{2}(x)}, \quad i=1,2 .
$$

This follows from a sequence of Lemmas. The involved arguments are similar to those of [1] where the linear Poisson structure case is considered.

Proof of Proposition 1

LEMMA 1. Let $\Gamma \in T_{n, 2}, p_{1}, p_{2} \in B_{\rho}(0)$. Then,

$$
\left|\hat{B}_{\Gamma}\left(p_{1}, p_{2}, x\right)\right| \leqslant M^{2 n-1} \rho^{n+1} .
$$

Proof. The estimate follows from that $\hat{B}_{\Gamma}$ is a $(n+1)$-homogeneous polynomial in the $p$ variables and an $n$-homogeneous polynomial in $\alpha$. Moreover, there are exactly $n-1$ derivatives differentiating the $\alpha$ 's.

LEMMA 2. $\left|T_{n, 2}\right| \leqslant(16 \mathrm{e})^{n} n$ !

Proof. The graphs in $T_{n, 2}$ are exactly the graphs in $G_{n, 2}$ such that $\Delta(\Gamma)$ is a tree, i.e., a graph without cycle. Thus, a graph $\Gamma \in T_{n, 2}$ may be described by specifying 
$\Delta(\Gamma), n^{n-2}$ choices, by giving each edge of $\Delta(\Gamma)$ an orientation, $2^{n-1}$ choices, by specifying for each vertex of $\Delta(\Gamma)$ if it has two outgoing edges going to $\{\overline{1}, \overline{2}\}$, just one or none, $4^{n}$ choices, and, at last, by giving the labeling 1 or 2 for each edge, $2^{n}$ choices. This procedure of counting is far from optimal. We count too many graphs and also graphs which are even not Kontsevich graphs. However, each graph in $T_{n, 2}$ is counted once. It gives us then the very crude estimate,

$$
\begin{aligned}
\left|T_{n, 2}\right| & \leqslant n^{n-2} 2^{n-1} 4^{n} 2^{n} \\
& \leqslant n^{n} 16^{n},
\end{aligned}
$$

which finishes the proof since $n^{n} \leqslant n ! e^{n}$.

DEFINITION 3. Let $\Gamma \in G_{n, 2}$. An aerial vertex $v \in V_{\Gamma}^{a}$ is called $a$ initial vertex if it has no incoming edge. A initial vertex $v \in V_{\Gamma}^{a}$ is called initial vertex of type 1 if one of $\gamma^{1}(v), \gamma^{2}(v)$ is in $\{\overline{1}, \overline{2}\}$ and the other is an aerial vertex. A initial vertex $v \in V_{\Gamma}^{a}$ is called initial vertex of type 2 if both $\gamma^{1}(v)$ and $\gamma^{2}(v)$ are aerial vertices. Trivially, there can be no initial vertex $v$ such that $\gamma^{1}(v), \gamma^{2}(v) \in\{\overline{1}, \overline{2}\}$.

LEMMA 3. Let be $\Gamma \in T_{n, 2}$. Then, $\Gamma$ has at least one initial vertex.

Proof. Suppose $\Gamma$ has no initial vertex. Let us construct the following sequence of vertices. Take $v_{0} \in V_{\Gamma}^{a}$. As $v_{0}$ is not a initial vertex, we may chose for $v_{1}$ any vertex such that $\left(v_{1}, v_{0}\right) \in E_{\Gamma}$. As there is no initial vertex, we can repeat this procedure infinitely many times. The result is an infinite sequence of vertices $\left\{v_{l}\right\}_{l \geq 0}$ of $V_{\Gamma}^{a}$. Now, as $\Gamma$ is a Kontsevich tree, there must be no $i, j$ such that $v_{i}=v_{j}$, this is a contradiction with the fact that $V_{\Gamma}^{a}$ is a finite set.

DEFINITION 4. Consider $\Gamma \in G_{n, m}$. Let $A \subset V_{\Gamma}$. We call $\Gamma_{/ A}$ the restriction of $\Gamma$ to $A$. It is the graph with vertex set $A$ and edges $E_{\Gamma} \cap(A \times A)$. We call $\Gamma_{(A)}$ the contraction of $\Gamma$ to $A$. It is the graph with vertex set $\left(V_{\Gamma} \backslash A\right) \sqcup\{*\}$ (the vertices of $A$ are contracted to a single vertex $*)$ and edges $(i, j) \in E_{\Gamma}$ where $i$ is replaced by the new vertex $*$ in $\Gamma_{(A)}$ if $i \in A$ and the same for $j$ (simple loops are deleted). Note that the resulting graphs might not be Kontsevich graphs.

LEMMA 4. Let $\Gamma \in T_{n, 2}$. Then, we have the following alternative:

1. There is a initial vertex of type $1, v \in V_{\Gamma}^{a}$, such that $\Gamma_{\mid V_{\Gamma} \backslash\{v\}} \in T_{n-1,2}$.

2. There is a initial vertex of type $2, v \in V_{\Gamma}^{a}$, such that the connected components of $\Gamma_{\mid V_{\Gamma} \backslash\{v\}}$ are two trees $\Gamma_{k} \in T_{k, 2}$ and $\Gamma_{l} \in T_{l, 2}$, for certain $k$ and $l$ such that $k+l=n-1$.

Proof. Take $\Gamma \in T_{n, 2}$. Consider $v$ an initial vertex in $\Gamma$. If $v$ is of type 1 , we are finished. If $v$ is not of type 1 , take another initial vertex. If this other vertex is of 


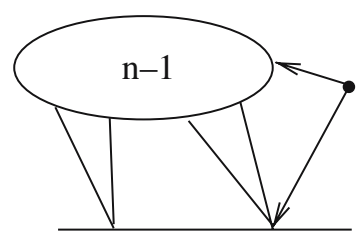

Case 1

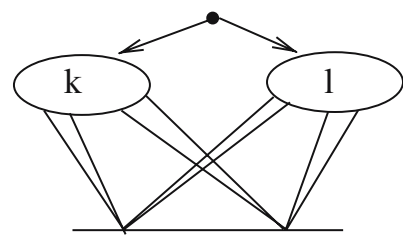

Case 2

Figure 2. Illustration of Lemma 4.

type 1 , we are finished. If there no initial vertex of type 1 , there must be at least one initial vertex $v$ of type 2 . The restriction of $\Delta(\Gamma)$ to $V_{\Gamma}^{a} \backslash\{v\}$ separate $\Delta(\Gamma)$ in two disjoint components $\Delta_{k}(\Gamma)$ and $\Delta_{l}(\Gamma)$. They are disjoint otherwise there would be a cycle in $\Delta(\Gamma)$. Now, it is easy to see that $\Delta_{k}$ is a tree with $k$ vertices and $\Delta_{l}$ a tree with $l$ vertices such that $k+l=n-1$ (if not $\Delta(\Gamma)$ is not a tree). Denote by $\Gamma_{k}\left(\right.$ resp. $\left.\Gamma_{l}\right)$ the tree $\Delta_{k}$ (reps. $\Delta_{l}$ ) to which we have added the legs back. Trivially, $\Gamma_{k}$ and $\Gamma_{l}$ are the connected components of $\Gamma_{\mid V_{\Gamma} \backslash\{v\}}$ and $\Gamma_{k} \in T_{k, 2}$, $\Gamma_{l} \in T_{l, 2}$.

LEMMA 5. For any $\Gamma \in T_{n, 2}$ and for any $\left(\phi_{1}^{1}, \phi_{1}^{2}, \ldots, \phi_{n}^{1}, \phi_{n}^{2}\right) \in\left(S^{1} \times S^{1}\right)^{n}$, there are at most $4^{n}$ elements $\left(z_{1}, \ldots, z_{n}\right) \in M_{n}$ such that

$$
\psi_{\Gamma}\left(z_{1}, \ldots, z_{n}\right)=\left(\phi_{1}^{1}, \phi_{1}^{2}, \ldots, \phi_{n}^{1}, \phi_{n}^{2}\right) .
$$

Proof. Fix two angles $\phi_{1}, \phi_{2}$ and two points $z_{1}, z_{2} \in \mathcal{H}$. There are at most $4 z \in$ $\mathcal{H}$ such that $\phi^{h}\left(z, z_{1}\right)=\phi_{1}$ and $\phi^{h}\left(z, z_{2}\right)=\phi_{2}$. Namely, we see from (7) that $z$ must be solution of the quadratic system of equations

$$
\mathrm{e}^{i \phi_{k}}\left(\bar{z}-z_{k}\right)\left(\bar{z}-\bar{z}_{k}\right)=\mathrm{e}^{-i \phi k}\left(z-z_{k}\right)\left(z-\bar{z}_{k}\right), \quad k=1,2,
$$

which is equivalent to ask that

$$
\operatorname{Im}\left(\mathrm{e}^{i \phi_{k}}\left(\bar{z}-z_{k}\right)\left(\bar{z}-\bar{z}_{k}\right)\right)=0, \quad k=1,2 .
$$

Thus, the Cartesian coordinates $(x, y)$ of $z$ must satisfies a system of two quadratic equations, which has at most four solutions. Similarly, the equation $\psi_{\Gamma}\left(z_{1}, \ldots, z_{n}\right)=\left(\phi_{1}^{1}, \phi_{1}^{2}, \ldots, \phi_{n}^{1}, \phi_{n}^{2}\right)$, is equivalent to a system of $2 n$ quadratic equations for the $2 n$ coordinates of $\left(z_{1}, \ldots, z_{n}\right)$. If non-degenerate, this system has at most $4^{n}$ solutions. However, there are graphs $\Gamma$ and angle values $\vec{\phi}$ for which this system is degenerate and hence the fiber $\psi_{\Gamma}^{-1}(\vec{\phi})$ infinite (consider for instance the Kontsevich graph $\Gamma \in G_{2,2}$ with one loop, and angle $\frac{\pi}{2}$ for the two legs, $\pi$ and $2 \pi$ for the aerial edges). We see, by induction, that it is never the case for the Kontsevich trees.

Namely, for $n=1$, clearly $\left|\psi_{\Gamma}^{-1}\left(\phi^{1}, \phi^{2}\right)\right| \leqslant 4$. Suppose now that the claim is true for all Kontsevich trees in $T_{u, 2}$ for $u=1, \ldots, n-1$. Then, Lemma 4, tells us that 
we have an alternative. If we are in the first case clearly by induction we have $\left|\phi_{\Gamma}^{-1}(\vec{\phi})\right| \leqslant 4^{n-1} 4=4^{n}$. In the second case, we have that, $\left|\phi_{\Gamma}^{-1}(\vec{\phi})\right| \leqslant 4^{k} 4^{l} 4=4^{n}$.

COROLLARY 1. For any $\Gamma \in T_{n, 2}$, we have that $\left|W_{\Gamma}\right| \leqslant 4^{n}$.

This corollary holds in fact for any graph in $G_{n, 2}$ thanks to an adapted version of Lemma 2.3 of [1].

Now, supposing without restricting the generality, that $M_{x}>1$, we can do the following estimate:

$$
\begin{aligned}
\left|\sum_{n=1}^{\infty} \epsilon^{n} \sum_{\Gamma \in T_{n, 2}} W_{\Gamma} \hat{B}_{\Gamma}\left(p_{1}, p_{2}, x\right)\right| & \leqslant \sum_{n=1}^{\infty} \frac{\epsilon^{n}}{n !}(16 \mathrm{e})^{n} n ! 4^{n} M^{2 n-1} \rho^{n+1} \\
& \leqslant \sum_{n=1}^{\infty} \epsilon^{n}\left(64 \mathrm{e} M^{2}\right)^{n} \rho^{n+1} \\
& \leqslant \frac{1}{64 \mathrm{e} M^{2}} \sum_{n=1}^{\infty} \epsilon^{n}, \quad p_{1}, p_{2} \in B_{\rho}(0),
\end{aligned}
$$

which gives the desired result.

\section{Comparison with Karasev Symmetric Solution}

\subsection{THE ANALYTICAL CASE}

We consider a Poisson manifold $M$ with a Poisson structure $\alpha$ such that in every chart $U$ for each $x \in U$ there exists a positive function $M_{U}(x)$ such that

$$
\left|\partial^{\beta} \alpha^{i j}(x)\right| \leqslant M_{U}(x)^{|\beta|+1},
$$

for all $i, j=1, \ldots, d$ and for all multi-index $\beta=\left(\beta_{1}, \ldots, \beta_{d}\right) \in \mathbb{N}^{d}$. We may assign to each chart $U$ the generating function,

$$
S_{\epsilon}\left(p_{1}, p_{2}, x\right)=x\left(p_{1}+p_{2}\right)+\sum_{n=1}^{\infty} \frac{\epsilon^{n}}{n !} \sum_{\Gamma \in T_{n, 2}} W_{\Gamma} \hat{B}_{\Gamma}\left(p_{1}, p_{2}, x\right)
$$

which is convergent, thanks to Proposition 1 , for $\left(p_{1}, p_{2}, x\right)$ taken in a suitable neighborhood of $B_{2}^{0}(U)$. In particular, there exists a neighborhood $\mathrm{G}_{\mathrm{U}}(\mathrm{S})$ such that $B_{1}^{0}(U) \subset \mathrm{G}_{\mathrm{U}}(\mathrm{S}) \subset T^{*} U$ and on which

$$
s(p, q)=\nabla_{p_{2}} S_{\epsilon}(p, 0, x) \text { and } t(p, q)=\nabla_{p_{1}} S_{\epsilon}(0, p, q),
$$

take their values in $U$ for all $(p, q) \in \mathrm{G}_{\mathrm{U}}(\mathrm{S})$. For convenience, set $B(x):=$ $2 \nabla_{p_{1}} \nabla_{p_{2}} S(0,0, x)=2 \epsilon \alpha(x)$. The following Proposition tells us that the points 
$x_{0}=s(p, q)$ and $x_{1}=t(p, q)$ are the end points of a curve $x(\tau)$ in $U$ satisfying a Hamilton-Poisson differential on $U$ with the linear Hamiltonian $l_{p}(x)=p x$ and initial condition $s(p, q)$.

PROPOSITION 2. Let $(\bar{p}, \bar{q}) \in \mathrm{G}_{\mathrm{U}}(\mathrm{S})$. Consider the Poisson equation

$$
\dot{x}^{i}(\tau)=B^{i j}(x(\tau)) \bar{p}_{j}, \quad x(0)=s(\bar{p}, \bar{q}) .
$$

Then,

$$
x(1)=t(\bar{p}, \bar{q}) .
$$

Moreover, the Hamilton system associated to $H(p, q)=-t^{*} l_{p}(p, q), l_{\bar{p}}(x)=\langle\bar{p}, x\rangle$, on $\mathrm{G}_{\mathrm{U}}(\mathrm{S})$ takes the point $(0, s(\bar{p}, \bar{q}))$ to the point $(\bar{p}, \bar{q})$ in time $t=1$.

Proof. Consider the Hamilton function $l_{\bar{p}}(x)=\langle\bar{p}, x\rangle$ on $U$. The vector fields $-\omega^{\sharp} t^{*} \mathrm{~d} l_{p}$ and $B^{\sharp} \mathrm{d} l_{\bar{p}}$ are $t$-related. Thus, the solution $(p(\tau), q(\tau))$ of the Hamilton system on $\mathrm{G}_{\mathrm{U}}(\mathrm{S})$,

$$
\begin{aligned}
& \dot{p}=\left\langle\frac{\partial}{\partial q} t(p, q), \bar{p}\right\rangle, \quad p(0)=0, \\
& \dot{q}=-\left\langle\frac{\partial}{\partial p} t(p, q), \bar{p}\right\rangle, \quad q(0)=s(\bar{p}, \bar{q})
\end{aligned}
$$

projects to the solution $x(\tau)=t(p(\tau), q(\tau))$ of the Poisson system $\dot{x}=B(x) p$, $x(0)=s(\bar{p}, \bar{q})$. Remark now that for all $q$ we have that

$$
\begin{aligned}
\left\langle\frac{\partial t}{\partial q}(t \bar{p}, q), \bar{p}\right\rangle & =\left\langle\nabla_{x} \nabla_{p_{1}} S_{\epsilon}(0, t \bar{p}, q), \bar{p}\right\rangle \\
& =\bar{p}+\sum_{i \geq 1} \epsilon^{i}\left\langle\nabla_{x} \nabla_{p_{1}} S_{\epsilon}^{(i)}(t \bar{p}, 0, q), \bar{p}\right\rangle \\
& =\bar{p},
\end{aligned}
$$

as for $i \geqslant 1, S_{\epsilon}^{(i)}$ is a sum over Kontsevich's trees. Thus, if we insert $p(\tau)=\bar{p} t$ in (9) and in (10), Equation (9) is trivially always satisfied and System (9)-(10) is reduced to a single equation on $q(\tau)$

$$
\dot{q}=-\left\langle\frac{\partial t}{\partial p}(\bar{p} t, q), \bar{p}\right\rangle, \quad q(0)=s(\bar{p}, \bar{q}) .
$$

For small $\bar{p}$, there always exists a unique solution for $q(\tau)$ and as for all $t$ we have that $s(p(\tau), q(\tau))=s(\bar{p}, \bar{q})$, then

$$
q(\tau)=Q(\bar{p} t, s(\bar{p}, \bar{q})) .
$$

Thus, $q(1)=Q(\bar{p}, s(\bar{p}, \bar{q}))=\bar{q}$ and we finally get that

$$
t(\bar{p}, \bar{q})=t(p(1), q(1))=x(1) .
$$


In [10] and [11], Karasev gives another local solution of the Lie system on $U$. Take a point $x \in U$ and a point $p \in \mathbb{R}^{d *}$ small enough so that the Poisson system

$$
\dot{x}^{i}=B^{i j}(x) p_{j}, \quad x(0)=x
$$

has a solution $x(\tau)$ defined for $t \in[-1,1]$. Consider the function

$$
Q^{\prime}(p, x)=\int_{0}^{1} x(\tau) \mathrm{d} \tau .
$$

If $p$ is small enough, we may invert this function. We define the source of the point $(p, q)$ as

$$
s^{\prime}(p, q)=x, \quad q=Q^{\prime}(p, x) .
$$

The target is defined as $t^{\prime}(p, x)=s^{\prime}(-p, x)$. It happens that $\left(s^{\prime}, t^{\prime}\right)$ is a solution of the Lie system in a neighborhood of the null section. This solution is called the symmetric solution. The next natural question to ask is how this symmetric solution compares to the one given by the generating function. For that purpose, we will use a Proposition proved in [10] and [11] by Karasev. Before stating the Proposition, let us give a definition.

DEFINITION 5. Let $(s, t)$ be a solution of the Lie system. Consider the following differential equation for $p(\tau)$,

$$
\dot{p}=\left\langle\frac{\partial t}{\partial q}\left(p, Q\left(p, x_{0}\right)\right), \bar{p}\right\rangle, \quad p(0)=0, \quad x_{0} \in U .
$$

Then, define the exponential map associated to $(s, t)$ as,

$$
\exp _{x_{0}}(\bar{p}):=p(1) .
$$

PROPOSITION 3 (KARASEV [11]). Let $(s, t)$ and $\left(s^{\prime}, t^{\prime}\right)$ be two solutions of the Lie system on $U$ and let $\exp$ and $\exp ^{\prime}$ be their associated exponential maps. Then, the transformation

$$
\begin{aligned}
& p^{\prime}=\exp _{x}^{\prime}\left(\exp _{x}^{-1}\right)(p) \\
& q^{\prime}=Q^{\prime}\left(p^{\prime}, x\right), \quad x=s(p, q),
\end{aligned}
$$

is a symplectic map and satisfies

$$
s^{\prime}\left(p^{\prime}, q^{\prime}\right)=s(p, q), \quad t^{\prime}\left(p^{\prime}, q^{\prime}\right)=t(p, q) .
$$

With the help of this Proposition, we may compare the symmetric solution and the one given by the generating function. In fact, in [11], Karasev proves that for the symmetric solution $\left(s^{\prime}, t^{\prime}\right)$ of the Lie system, $\exp _{x}^{\prime}(p)=p$. As for the one given by the generating function, we get from the proof of Proposition 2 also that $\exp _{x}(p)=p$. 
PROPOSITION 4 (COMPARISON). The solution $(s, t)$ of the Lie system given by the symplectic groupoid generating function is exactly the symmetric solution, i.e., if we consider the Poisson system

$$
\dot{x}^{i}(\tau)=B^{i j}(x) p_{j}, \quad x(0)=x,
$$

then,

$$
\begin{aligned}
Q(p, x) & =\int_{0}^{1} x(\tau) \mathrm{d} \tau=\nabla_{p_{2}} S(-p, p, x), \\
Q(p, s(p, q)) & =q, \quad s(p, Q(p, x))=x .
\end{aligned}
$$

Proof. Consider the symmetric solution $\left(s^{\prime}, t^{\prime}\right)$. In this case, by Proposition 3, the Karasev transformation relating $(s, t)$ and $\left(s^{\prime}, t^{\prime}\right)$ takes the form

$$
\begin{aligned}
p^{\prime} & =p, \\
q^{\prime} & =Q^{\prime}(p, s(p, q)) .
\end{aligned}
$$

This transformation is also symplectic. This imposes on the transformation that

$$
\frac{\partial q^{\prime}}{\partial q}=\mathrm{id} \quad \text { and } \quad \frac{\partial q^{\prime}}{\partial p}=\left(\frac{\partial q^{\prime}}{\partial p}\right)^{*} .
$$

Hence,

$$
\frac{\partial q^{\prime}}{\partial q}=\frac{\partial Q^{\prime}}{\partial x}(p, x) \frac{\partial s}{\partial q}(p, q)=\mathrm{id} .
$$

As we also have that

$$
\frac{\partial Q}{\partial x}(p, x) \frac{\partial s}{\partial q}(p, q)=\mathrm{id},
$$

then,

$$
\frac{\partial Q^{\prime}}{\partial x}(p, x)=\frac{\partial Q}{\partial x}(p, x),
$$

for all $p, x$. Thus,

$$
Q^{\prime}(p, x)=Q(p, x)+C(p),
$$

and the Karasev transformation becomes

$$
\begin{aligned}
& p^{\prime}=p, \\
& q^{\prime}=Q(p, s(p, q))+C(p)=q+C(p) .
\end{aligned}
$$

Now,

$$
\frac{\partial q^{\prime}}{\partial p}=\left(\frac{\partial q^{\prime}}{\partial p}\right)^{*},
$$


implies that

$$
\frac{\partial C}{\partial p}(p)=\left(\frac{\partial C}{\partial p}(p)\right)^{*}
$$

Hence, by Stokes, there exists a function $f: \mathbb{R}^{d *} \rightarrow \mathbb{R}$ such that $C(p)=\nabla f(p)$. Now, as we have for all $p, x$ and $\lambda \in \mathbb{R}$ that,

$$
\langle Q(\lambda p, x), p\rangle=\langle x, p\rangle, \quad\left\langle Q^{\prime}(\lambda p, x), p\right\rangle=\langle x, p\rangle .
$$

Equation (12) gives that

$$
\langle C(\lambda p), p\rangle=\langle\nabla f(\lambda p), p\rangle=0 .
$$

As

$$
\frac{\mathrm{d}}{\mathrm{d} \lambda} f(\lambda p)=\langle\nabla f(\lambda p), p\rangle=0,
$$

we get that $f(\lambda p)=f(0)$, i.e., $f$ is constant and finally

$$
\nabla f(p)=c(p)=0
$$

which completes the proof.

\subsection{THE SMOOTH CASE AND FORMAL SYMPLECTIC GROUPOIDS}

If the Poisson structure $\alpha$ on $U$ is only smooth, we can not guarantee that the generating function $S_{\epsilon}$ converges anymore. The induced symplectic groupoid is then only a formal one. On the other hand, we may still consider the KarasevMaslov construction for the formal Poisson structure $B=2 \epsilon \alpha$, where $\epsilon$ is a formal parameter. This leads also to a formal symplectic groupoid which, we will see, coincides with the one coming from the generating function exactly as in the analytical case. We will only show that the formal source of both formal symplectic groupoids are equal as all the other structures may be recovered from the source.

Let us denote by $\bar{s}$ the formal source coming from the Karasev-Maslov construction and by $s$ the one coming from the generating function. The formal solution $X_{\epsilon}(t)$ of the Poisson system $X_{\epsilon}(t)=B(X(t)) p$ is then a formal power series of the form $X_{\epsilon}(t)=x+2(\epsilon t) \alpha(x) p+\mathcal{O}\left((\epsilon t)^{2}\right)$. The first order computation for the source of Karasev-Maslov yields $\bar{s}^{l}(p, x)=x^{l}+\epsilon \alpha^{k l}(x) p_{k}+\mathcal{O}\left(\epsilon^{2}\right)$. Obviously, $\bar{s}(p, x)=x+\epsilon \bar{s}_{1}(p, x)+\epsilon^{2} \bar{s}_{2}(p, x)+\cdots$ is a formal solution of the formal Lie system for $B$ on $U$, which means in particular that we have at order $n$ that

$$
\frac{\partial \bar{s}_{n}^{j}}{\partial p_{i}}-\frac{\partial \bar{s}_{n}^{i}}{\partial p_{j}}=H_{n}\left(\bar{s}_{1}, \ldots, \bar{s}_{n-1}\right)
$$


where $H_{n}$ is a certain function depending only on $\bar{s}_{1}, \ldots, \bar{s}_{n-1}$. Suppose now by induction that $s_{k}=\bar{s}_{k}$ for $k=1, \ldots, n-1$. As $s$ also satisfies the formal Lie system (see [2]), we have that the difference $g_{n}:=s_{n}-\bar{s}_{n}$ satisfies

$$
\frac{\partial g_{n}^{j}}{\partial p_{i}}-\frac{\partial g_{n}^{i}}{\partial p_{j}}=0
$$

This implies by Stokes that there is a function $f_{n}(p, x)$ such that $\nabla_{p} f_{n}(p, x)=g_{n}$. Now we have that

$$
\frac{\mathrm{d}}{\mathrm{d} \lambda} f_{n}(\lambda p, x)=\left\langle\nabla_{p} f_{n}(\lambda p, x), p\right\rangle=\left\langle s_{n}(\lambda p, x), p\right\rangle-\left\langle\bar{s}_{n}(\lambda p, x), p\right\rangle .
$$

One sees easily that $\langle s(\lambda p, x), p\rangle=\langle\bar{s}(\lambda p, x), p\rangle=\langle x, p\rangle$ and, consequently, that $f_{n}(p, x)$ does not depend on $p$. Thus $g_{n}=s_{n}-\bar{s}_{n}=0$. As the formal source coming from the Karasev-Maslov construction and the one coming from the generating function have both the same first order term, they are equal.

Given a smooth Poisson structure $\alpha$, we may also consider the Poisson sigma model of Cattaneo-Felder (see [4]) to get a formal symplectic groupoid. The idea is to replace the Gauss law by its formal version

$$
\dot{X}(t)=\epsilon \alpha(X(t)) \eta(t),
$$

as well as the symmetries by their formal version. An element in the formal symplectic groupoid is an equivalence class of perturbative solutions

$$
\left[\left(X_{0}+\epsilon X_{1}+\cdots, \eta_{0}+\epsilon \eta_{1}+\cdots\right)\right] .
$$

The comparison with the formal symplectic groupoid coming from the generating function is more involved and will be the subject of a subsequent work. Note also that the approach of Crainic and Fernandes (see [5]) may also lead to formal symplectic groupoids.

\section{Acknowledgements}

The author thanks Giovanni Felder and Alberto S. Cattaneo for their help and encouraging support as well as Alan Weinstein for useful suggestions. B.D. acknowledges partial support of SNF Grant No. 21-65213.01.

\section{References}

1. Andler, M., Dvorsky, A., Sahi, S.: Kontsevich quantization and invariant distributions on Lie groups. Ann. Sci. école Norm. Sup. (4) 35(3), 371-390, math.QA/9910104 (2002)

2. Cattaneo, A.S., Dherin, B., Felder, G.: Formal symplectic groupoid. Comm. Math. Phys. 253(3), 645-674, math.SG/0312380 (2005)

3. Cattaneo, A.S., Dherin, B., Felder, G.: Formal Lagrangian operad. math.SG/0505051 
4. Cattaneo, A.S., Giovanni, F.: Poisson sigma models and symplectic groupoids. Quantization of singular symplectic quotients, pp. 61-93, Progr. Math., vol. 198, Birkhäuser, Basel (2001)

5. Crainic, M., Fernandes, R.: Integrability of Poisson brackets. J. Diff. Geom. 66(1), 71-137 (2004)

6. Coste, A., Dazord, P., Weinstein, A.: Groupoïdes symplectiques. (French) [Symplectic groupoids] Publications du Département de Mathématiques. Nouvelle Série. A, vol. 2, i-ii, pp. 1-62, Publ. Dép. Math. Nouvelle Sér. A, 87-2, Univ. Claude-Bernard, Lyon (1987)

7. de M. Rios, P., Ozorio de Almeida, A.: A variational principle for actions on symmetric symplectic spaces. J. Geom. Phys. 51(4), 404 441 (2004)

8. Dherin, B.: Star products and symplectic groupoids. Diss. ETH Zürich. No. 15720, http://www.math.unizh.ch/reports/08_05.pdf (2004)

9. Karabegov, A.V.: Formal symplectic groupoid of a deformation quantization. math.QA/0408007 41 pp. QA

10. Karasev, M.V.: Analogues of objects of the theory of Lie groups for nonlinear Poisson brackets. (Russian) Izv. Akad. Nauk SSSR Ser. Mat. 50(3), 508-538, 638 (1986)

11. Karasev, M.V.: The Maslov quantization conditions in higher cohomology and analogs of notions developed in Lie theory for canonical fibre bundles of symplectic manifolds. II Selecta Mathematica Sovietica. vol. 8(3), 235-257 (1989)

12. Karasev, M.V., Maslov, V.P.: Nonlinear Poisson brackets. Geometry and quantization. Translated from the Russian by A. Sossinsky [A.B. Sosinskii] and M. Shishkova. Translations of Mathematical Monographs, 119. American Mathematical Society, Providence, RI, xii+366 pp., ISBN: 0-8218-4596-9 (1993)

13. Kontsevich, M.: Deformation quantization of Poisson manifolds, I. q-alg/9709040 (1997)

14. Weinstein, A.: The local structure of Poisson manifolds. J. Diff. Geom. 18, 523-557 (1983)

15. Weinstein, A.: Symplectic groupoids and Poisson manifolds. Bull. Am. Math. Soc. (N.S.) 16(1), 101-104 (1987) 